\title{
Theoretical Semiempirical Study of the Nitrone (Anticancer Drug) Interaction with Fullerene C60 (as Delivery)
}

\author{
M.J. Al-AnBer ${ }^{a, *}$, A.H. Al-MowAli ${ }^{b}$ And A.M. Ali ${ }^{a}$ \\ ${ }^{a}$ Department of Physics, College of Science, Basrah University, Karmet Ali, Basrah, Iraq \\ ${ }^{b}$ Department of Chemistry, College of Science, Basrah University, Karmet Ali, Basrah, Iraq
}

(Received May 9, 2013; in final form March 5, 2014)

\begin{abstract}
Modelling of the quantum interaction properties of nitrone on the fullerene $\mathrm{C} 60$ has been investigated by the use of PM3 calculations. It has been found that the interaction potential of the nitrone with the fullerene C60 led to stable complexes when the fullerene reacted with the carbon atom $\left(C^{1}\right.$-centered) and metastable conformations with carbon atom $\left(C^{2}\right.$-centered). We have studied the effect of two rotation characteristics (nitrone and $\mathrm{NOCH}_{2}$ group) on the binding between the fullerene $\mathrm{C} 60$ with the nitrone. Our results suggested that the binding energy is lower as the nitrone rotation increases and the $\mathrm{NOCH}_{2}$ group rotation showed more effect. The fullerene C60 may be able to do more linking with molecules of nitrone.
\end{abstract}

DOI: 10.12693 /APhysPolA.126.845

PACS: 87.15.Aa

\section{Introduction}

Fullerenes are molecules composed entirely of carbon, in the form of a hollow sphere, and similar in structure to graphite, which is composed of stacked graphene sheets of linked hexagonal rings, but they may also contain pentagonal rings, too. The fullerenes unique properties have attracted considerable interest in many fields for example biomedical applications. Available data clearly shows that the $\mathrm{C} 60$ has no acute or sub-acute toxicity in a large variety of living organisms [1]. The fullerene was prepared in 1985 by Smalley and co-workers at Rice University; its structure was identified some five years earlier by Iijima [2]. Fullerenes have been found to occur in nature [3]. More recently, fullerenes have been detected in outer space and according to astronomer Stanghellini, "It's possible that Bucky balls from outer space provided seeds for life on Earth" [4, 5]. The characteristics and behaviour of nanomaterial are a new field of science, being limited to nanoscale dimensions $(1-100 \mathrm{~nm})$. The nanostructures have a quantum nature due to their atomic and molecular size. Thus, the prediction and understanding of these nanomaterials must be based on experimental and theoretical research. A question is how the experiments can be approached at the atomic level to do nanomeasurements? The fullerene $\mathrm{C} 60$ has special thermal, mechanical and electrical properties and the ability to be applied at atomic levels, this makes them promising structures for working in a wide range of applications. Many of these applications are in biology and potential medicinal use such as binding specific antibiotics to the structure to target resistant bacteria and even tar-

\footnotetext{
${ }^{*}$ corresponding author; e-mail:

mohanned.mohammed@uobasrah. edu. iq
}

get certain cancer cells such as melanoma [6]. Fullerenes can be made to be absorbed by HeLa cells. The C60 derivatives can be delivered to the cells by using the functional groups L-phenylalanine, folic acid and L-arginine among others [7, 8]. There are few calculations that have been made by using ab initio quantum methods applied to fullerenes $[9,10]$. In spite of the wide applications for fullerene $\mathrm{C} 60$ the theoretical studies of the interaction mechanism with biomolecules are few, not many researchers have studied the interaction of an amino acid with nanomaterials $[11,12]$.

In this work, we studied the interaction of the nitrone on the surface of the fullerene C60. Then we examined this interaction as a function of nitrone rotates and the $\mathrm{NOCH}_{2}$ group rotates. Finally we tried to investigate the ability of linking many molecules of nitrone on the fullerene surface.

\section{Computational details}

Theoretical calculations can be used to bridge gaps in understanding experimental results. In many cases, the results of the experimental methods are unable to accurately describe small systems of complex biomolecule. The methods of molecular quantum mechanics can be used to investigate properties beyond the scope of current crystallographic methods. The molecular quantum techniques allow us to study optical, magnetic and electronic properties that are not easily measured experimentally Molecular quantum mechanics provide the interaction energies that are not provided by X-ray and nuclear magnetic resonance (NMR) experiments. The theoretical methods can be used to further investigate and predict the physical and chemical nature of hydrogen bonding interactions. To investigate the structural and electronic properties of fullerene molecules decorated with the nitrone, we used PM3 (parameterizes method). PM3 developed by Stewart $[13,14]$, is a reparameterization of 
AM1, which is based on the neglect of diatomic differential overlap (NDDO) approximation. NDDO retains all one-center differential overlap terms when Coulomb and exchange integrals are computed. PM3 differs from AM1 only in the values of the parameters. The parameters for PM3 were derived by comparing a much larger number and wider variety of experimental versus computed molecular properties. Typically, non-bonded interactions are less repulsive in PM3 than in AM1. PM3 is primarily used for organic molecules, but is also parameterized for many main group elements. For these reasons it gives better results than MNDO, MNDO/d and AM1 methods, even though those methods are generally more accurate. The problem that arises is how to perform an accurate calculation for a nanosized system without ending in a prohibitively large computation. The resolution of PM3, as implemented in the HyperChem Release 7.52 for Windows Molecular Modeling System program package (http://wwwhyper.com/), was employed for the geometry optimizations.

\section{Results and discussion}

Initially, we expected two isomers for the molecule of nitrone. For our investigation, it was important to determine the most stable isomer. The molecule of nitrone

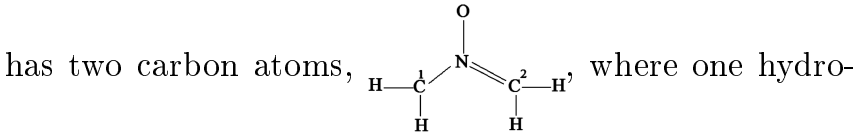
gen atom is abstracted from the carbon atom number 1 to obtain the $C^{1}$-centered (see Fig. 1). Then one hydro-

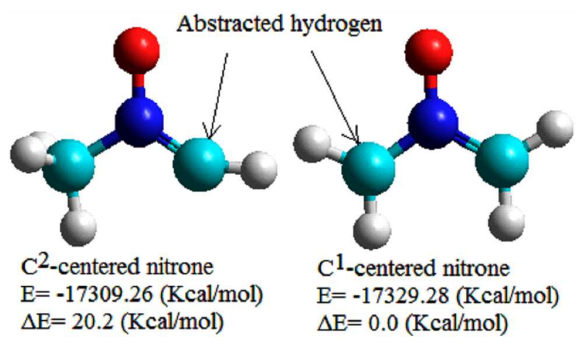

Fig. 1. The most stable isomers of the nitrone and their relative stability, $\Delta E$.

gen atom is abstracted from the carbon atom number 2 to obtain the $C^{2}$-centered. So there are two isomers with centered $C^{1}$ and $C^{2}$-centered, respectively. According to the relative stability, $\Delta E=E_{C 1-\text { nitrone }}-E_{C 2-\text { nitrone }}$ it was found that the $C^{1}$-centered nitrone is favored over the $C^{2}$-centered ones by $20.2 \mathrm{kcal} / \mathrm{mol}$, employing the PM3 method. Then, we calculated the interaction, binding energy $(B E), B E=E_{\text {nitrone+fullerene }}$ $\left(E_{\text {nitrone }}+E_{\text {fullerene }}\right)$, between the two centered nitrones with fullerene molecules. We found that upon reaction of nitrone with the fullerene, the nitrone forms stable complexes when the fullerene reacts with the nitrone's carbon atom $\left(C^{1}\right.$-centered) and metastable conformations with
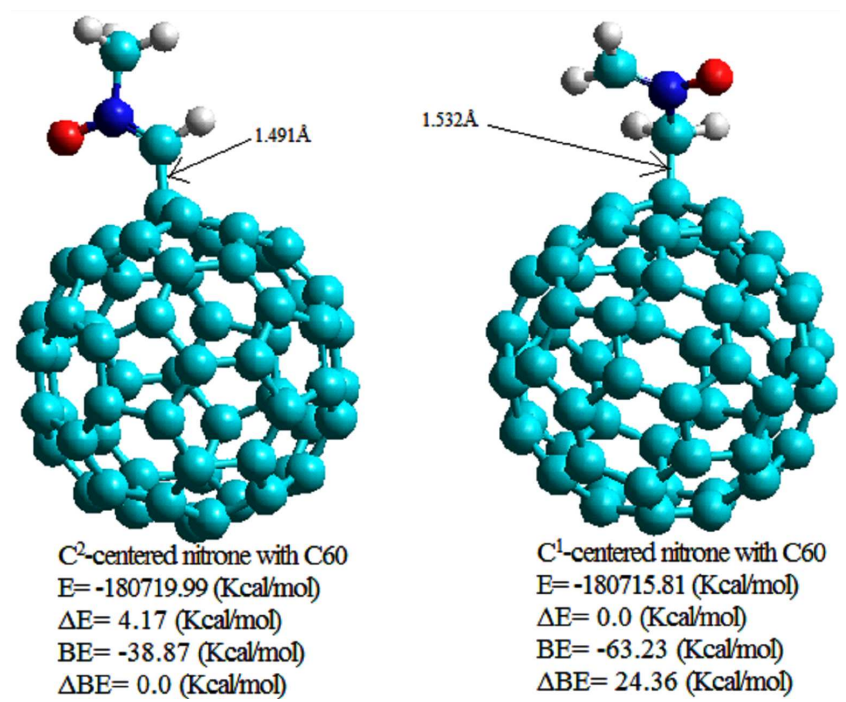

Fig. 2. Geometry optimized structures of nitronefullerene.

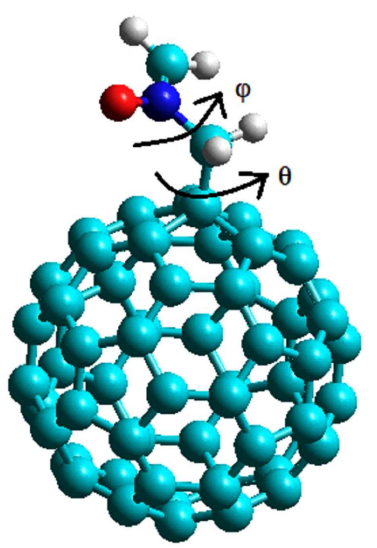

Fig. 3. Displays the direction of nitrone rotation about the bond that link nitrone with fullerene $\theta$ and the rotation of $\mathrm{NOCH}_{2}$ group $\varphi$.

the carbon atom in $C^{2}$-centered nitrone as shown in Fig. 2 along with their relative binding energies $\triangle B E$ and relative stabilities, $\Delta E$ The bond length, which connects the nitrone with fullerene, is dependent on the centered nitrone, the length of the bond in issues of the $C^{2}$-centered is lower than the $C^{1}$-centered.

The $C^{1}$-centered radical has more ability to connect with fullerene in comparison with the $C^{2}$-centered radical, but the relative stability, $\Delta E=4.17 \mathrm{kcal} / \mathrm{mol}$ not as much. The $C^{1}$-centered nitrone will be adopted in the present study. Our next step is the examination of the interaction between the $C^{1}$-centered radical nitrone with fullerene as a function of the nitrone rotates, with angle $\theta$, about the bond which connects it with the fullerene, with rotate step $10^{\circ}$, see Fig. 3 .

The effect of the increase in the rotation angle, $\theta$, on the relative stability, $\Delta E$, for the $C^{1}$-centered nitrone- 


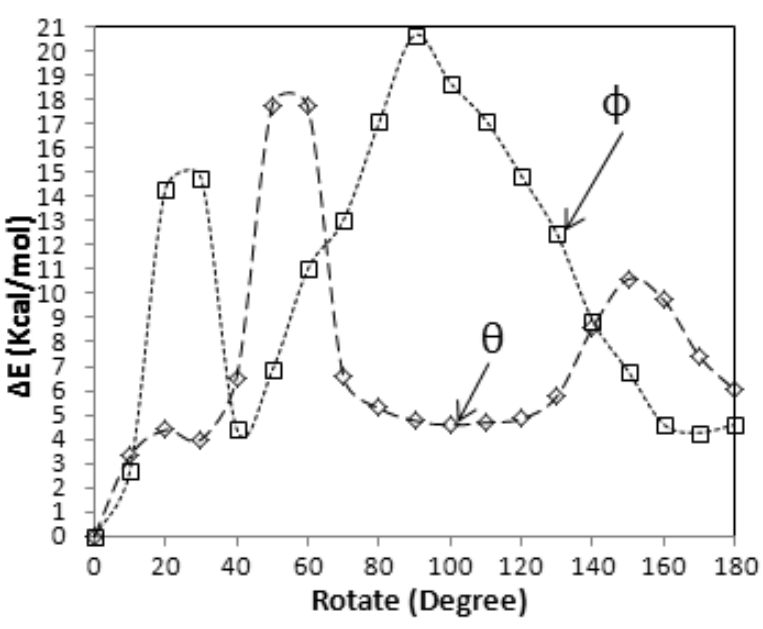

Fig. 4. The relative stability $\Delta E$ of the $C^{1}$-centered nitrone with fullerenes as a function of the rotation angle $\theta$ and $\varphi$.

C60 is shown in Fig. 4. Increasing in the nitrone radical's rotation about the bond that linked it with fullerene will decrease the relative stability of the $C^{1}$-centered nitroneC60. This situation shows the mechanism of lowering binding force between the nitrone and the fullerene due to the angle $\theta$. Figure 4 shows there were fluctuations in the relative stability with the increase of $\theta$. Rotating the nitrone with an angle more than $40^{\circ}$ will lower the interaction force rapidly. The required energy for this rotation is minimal. For this reason we expect, that it is not hard to break this bond there is a decrease at $150^{\circ}$, but it is not comparable with the former. The second step is about the interaction of the $C^{1}$-centered radical with fullerene as a function of the $\mathrm{NOCH}_{2}$ group rotation, with angle $\varphi$, about the single bond between the nitrogen-carbon in the nitrone, with rotating step $10^{\circ}$, see Fig. 3. The effects of an increase in the rotation of $\mathrm{NOCH}_{2}$ group on the relative stability $(\Delta E)$ for the $C^{1}$-centered nitrone-C60 are shown in Fig. 4. Due to the $\mathrm{NOCH}_{2}$ group rotates, the relative stability of the nitron-C60 system shows it to be decreasing. There are two interesting rotation angles, $10^{\circ}<\varphi<40^{\circ}$ and $50^{\circ}<$ $\varphi<160^{\circ}$, with a rotation angle more than $\varphi=10^{\circ}$, there is a rapid lowering in the relative stability for the nitrone-C60. According to Fig. 4, the rotation of $\mathrm{NOCH}_{2}$ group is more influential on the relative stability for the $C^{1}$-centered nitrone-C60, in comparison with the former.

For the final step, we tried to examine the ability of linking more than one $C^{1}$-centered nitrone on the surface of Fullerene $\mathrm{C} 60$ we linked another $C^{1}$-centered nitrone on the fullerene C60, see Fig. 5a. Where we linked the second $C^{1}$-centered nitrone at position 1 we optimized the system. In order to examine the best position to link the second nitrone, according to the first one, we tried the other positions $2,3 \ldots 8$.

At each new position, we re-optimized the system again. Fig. 6 shows the results of the relative stability as

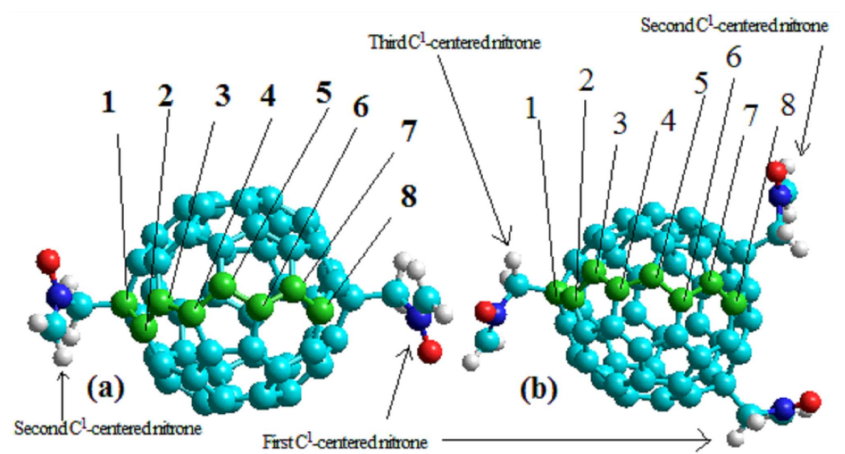

Fig. 5. (a) The locations of linking the second radical of nitrone with fullerenes, (b) add the third nitrone.

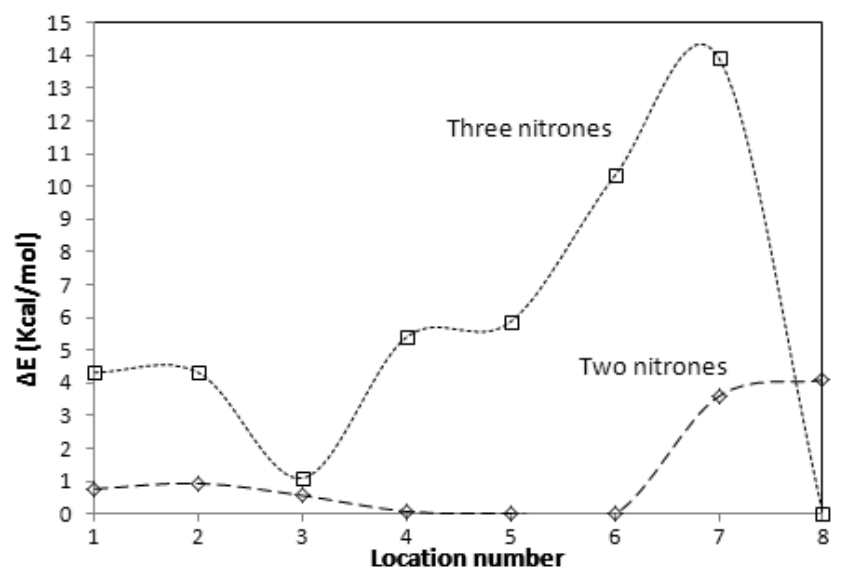

Fig. 6. The results of relative stability as a function of the change of the second then the third radical of nitrone locations on the surface of fullerene.

a function of the different positions for the second nitrone on the fullerene surface. The second $C^{1}$-centered nitrone preferred to link at the position 5 and 6 on the fullerene surface. In order to investigate the ability of linking more than two $C^{1}$-centered nitrone on the surface of fullerene we linked the third $C^{1}$-centered nitrone on the fullerene C60, see Fig. 5b according to the linking results of two $C^{1}$-centered nitrones on the fullerene surface, see Fig. 6 , where the second nitrone preferred to link at position 5 , thus we will adopt this issue, in order to examine the ability of the addition of the third nitrone. We linked the third $C^{1}$-centered nitrone at position 1 , and then we re-optimized the system again. In order to examine the best position to link the third nitrone, according to the two other $C^{1}$-centered nitrones, we tried the other positions $2,3 \ldots 8$. At each new position, we re-optimized the system again. Figure 6 shows the results of the relative stability as a function of the different positions for the third nitrone on the fullerene surface, where the position 3 is the best location to link the third $C^{1}$-centered nitrone on the fullerene surface. The fullerene C60 may be able to do many linkages with nitrone, in comparison with the carbon nanotubes (CNTs) [15]. We think the 
fullerene can be used in drug-delivery more suitably than the carbon nanotubes. The stability would decrease to become critical, when the third $C^{1}$-centered nitrone was linking at position 8 , where the third $C^{1}$-centered nitrone becomes non-linking on the fullerene surface.

\section{Conclusions}

We have performed PM3 calculations on the structural properties of fullerene $\mathrm{C} 60$ upon adsorption of various nitrones among these two isomers, the $C^{1}$-centered nitrone forms stable complexes with fullerene. The results of the rotation (nitrone and $\mathrm{NOCH}_{2}$ group, respectively) on the relative stability of nitrone-fullerene show a decrease as rotation increases for some rotation regions and the rotation of $\mathrm{NOCH}_{2}$ group is more influential on the relative stability for the $C^{1}$-centered nitrone-C60, in comparison with the former. The fullerene $\mathrm{C} 60$ may be able to do many linkages with nitrones. The fullerene can be used in drug-delivery being more suitable than the carbon nanotubes.

\section{References}

[1] J. Kolosnjaj, H. Szwarc, F. Moussa, Adv. Exp. Med. Biol. 620, 168 (2007).

[2] S. Iijima, J. Cryst. Growth 50, 675 (1980).
[3] P. Buseck, S. Tsipursky, R. Hettich, Science 257, 215 (1992).

[4] J. Cami, J. Bernard-Salas, E. Peeters, S. Malek, Science 329, 1180 (2010).

[5] D. García-Hernández, A. Manchado, P. García-Lario, L. Stanghellini, E. Villaver, R. Shaw, R. Szczerba, J. Perea-Calderón, Astrophys. J. Lett. 724, L39 (2010).

[6] G.P. Tegos, T.N. Demidova, D. Arcila-Lopez, H. Lee, T. Wharton, H. Gali, M. R. Hamblin, Chem. Biol. 12, 1127 (2005).

[7] S.B. Brown, Lancet Oncology 5, 497 (2004).

[8] P. Mroz, Free Radical Biol. Med. 43, 711 (2007).

[9] A.F. Jalbout, A.J. Hameed, I. Jimenez-Fabian, M. Ibrahim, A. de Leon, J. Org. Chem. 693, 216 (2008).

[10] M. Arndt, O. Nairz, J. Vos-Andreae, C. Keller, G. van der Zouw, A. Zeilinger, Nature 401, 680 (1999).

[11] A. Mavrandonakis, S.C. Farantos, G.E. Froudakis, J. Phys. Chem. B 110, 6048 (2006).

[12] M. Al-Anber, J. Macro. Sci. B 50, 2481 (2011).

[13] J.J.P. Stewart, J. Comp. Chem. 10, 209 (1989).

[14] J.J.P. Stewart, J. Comp. Chem. 10, 221 (1989).

[15] M. Al-Anber, A. Ali, S. Resan, A. Al-Mouali, Int. J. Green Nanotech. 3, 238 (2011). 